Archives of Clinical and Medical Case Reports

doi: $10.26502 /$ acmcr. 96550035

fortune

Volume 2, Issue 5

Case Report

\title{
Carotid Artery Dissection Causing an Isolated Hypoglossal Nerve Palsy
}

\section{Muzzammil Ali*, Yatin Sardana}

Acute Medical Unit, Solihull Hospital, Heart of England Foundation Trust, Solihull, West Midlands, England

*Corresponding Author: Dr. Muzzammil Ali, Acute Medical Unit, Solihull Hospital, Heart of England Foundation Trust, Solihull, West Midlands, England, Tel: +07907822431; E-mail: muzzammil.ali@nhs.net

Received: 25 September 2018; Accepted: 02 October 2018; Published: 05 October 2018

\begin{abstract}
Carotid Artery Dissection (CAD) typically presents with an acute onset of neck pain and headache. It can occur spontaneously or secondary to neck trauma. Neck trauma can be major following a road traffic accident or assault, or can be minor, for example, through coughing, sneezing or hiccups. There is also a postulated link between CAD and upper respiratory tract infections. Here we describe an unusual case of CAD presenting as a hypoglossal nerve palsy which was preceded by a coryzal illness. It highlights the importance of maintaining a high index of suspicion in patients with acute focal neurology. The early diagnosis and management of CAD helps to prevent complications such as ischemic stroke or loss of vision.
\end{abstract}

Keywords: CT-Angiogram; Embolization; Thrombus

\section{Case Report}

A 49-year-old gentleman was referred by his GP to the acute medical unit. He presented with a four day history of a sudden onset of left posterior neck pain and left sided ear ache. He then developed a gradual onset of slurred speech and difficulty in moving his tongue. He stated that his "tongue feels enlarged." There was no history of major trauma or excessive exercise. Seven days prior to this hospital presentation, he complained of having developed a mild coryzal illness with an occasional dry cough. 
On examination he displayed features consistent with an isolated left hypoglossal nerve (CNXII) palsy- left sided tongue deviation, mild left sided tongue atrophy and mild dysarthria (Figure 1).
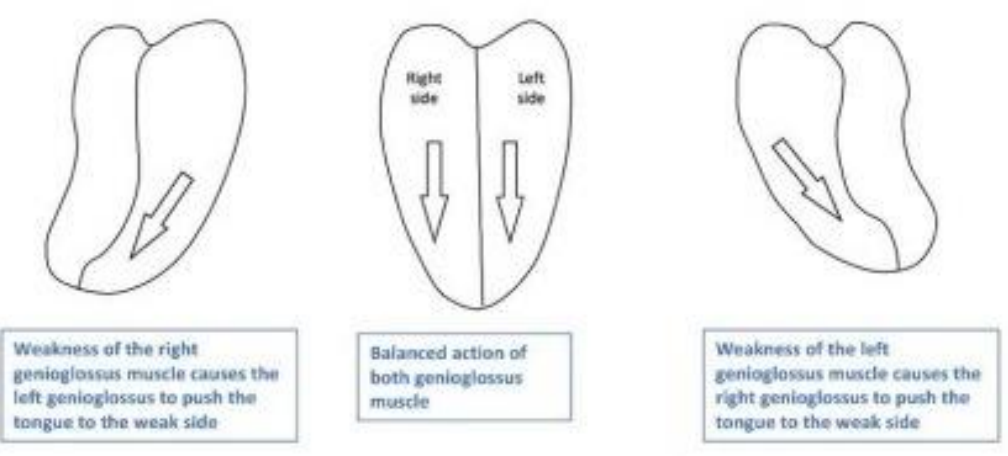

Figure 1: The CNXII supplies motor function to the tongue. CNXII palsy is manifested as tongue deviation toward the side of injury.

There was no other neurological deficit identified. His blood tests were unremarkable and his ECG showed normal sinus rhythm. He was reviewed by the inpatient neurology team who recommended brain imaging to rule out a vascular and/or intracranial pathology. An MRI-Head showed diminished flow within the left internal carotid artery. A subsequent CT-angiogram of the aortic arch and carotids showed features consistent with a left cervical internal carotid artery dissection (CAD) (Figures 2-4).

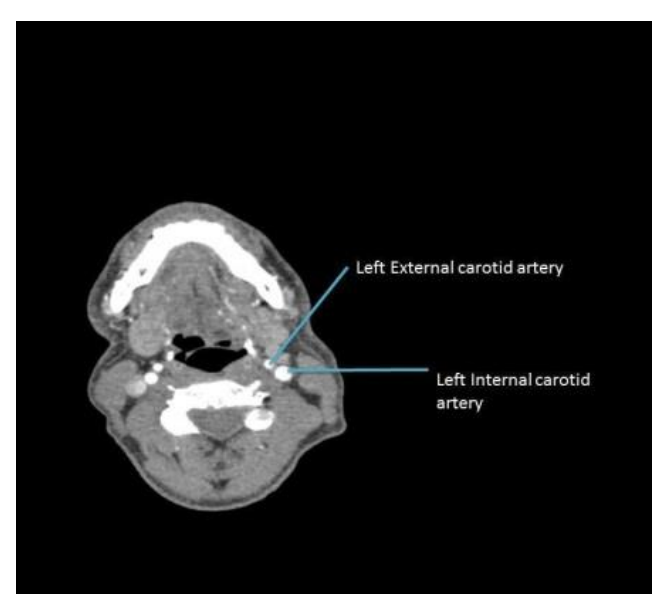

Figure 2: CT-Angiogram showing patent left internal and external carotid arteries at the level of the lower cervical spine. 


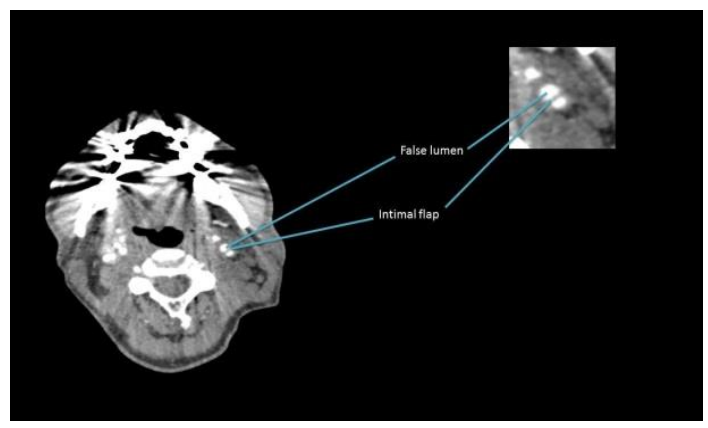

Figure 3: CT-Angiogram showing a left CAD at the level of the upper cervical spine. There is the formation of an intimal flap separating the true and false lumens. There is also a small diverticulum/focal saccular aneurysm projecting from this artery at this level.

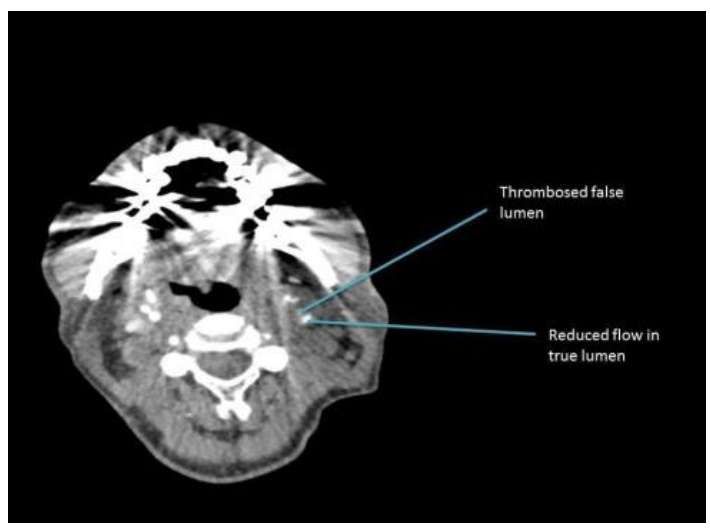

Figure 4: CT-Angiogram showing a thrombosed false lumen (intramural haematoma).

The Royal College of Physicians recommends using anticoagulation or antiplatelet therapy for three months in managing CAD [1]. This patient was started on aspirin and his symptoms began to improve as his antiplatelet course continued. CAD occurs due to a tear being formed in the tunica intima, resulting in an intramural haematoma and the creation of a false lumen (Figure 5).

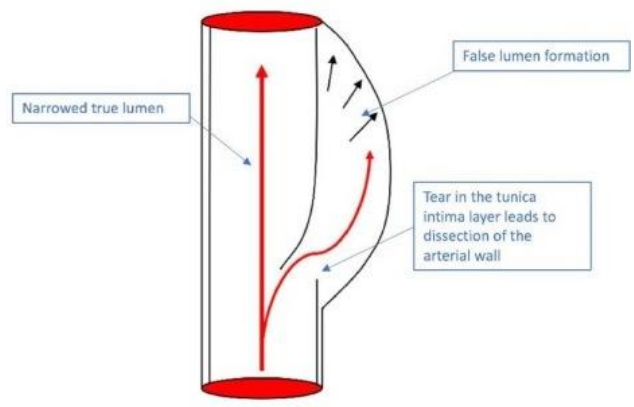

Figure 5: CAD occurs due to a tear being formed in the tunica intima, resulting in an intramural haematoma and the creation of a false lumen. 
Thrombus formation (Figure 6,7) and distal embolization (Figure 8) may lead to an ischaemic stroke [2].

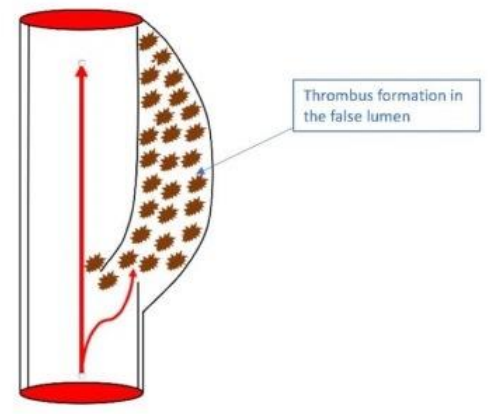

Figure 6: Thrombus formation within the false lumen.

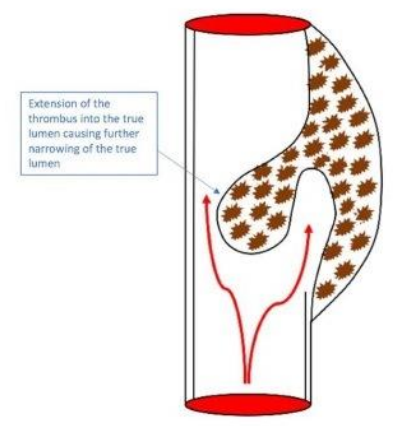

Figure 7: Thrombus formation and a subsequent increase in size within the false lumen can cause a reduction in blood flow within the true lumen.

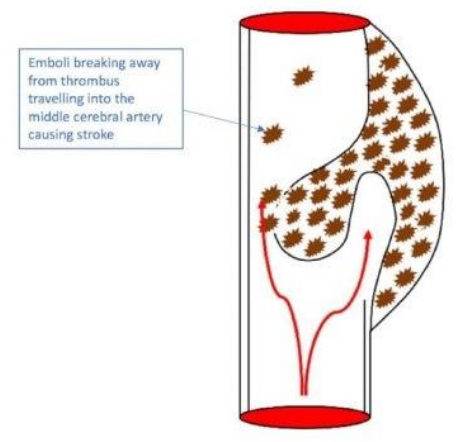

Figure 8: Emboli can break off of an intramural thrombus. They can travel to the Circle of Willis and manifest as an ischamic stroke. 
Alternatively, there may be aneurysmal dilatation which may result in the compression of neighboring structures [2]. CNXII for example passes inferiorly to the angle of the mandible, crossing the internal and external carotid arteries before moving anteriorly to enter the tongue (Figure 9).

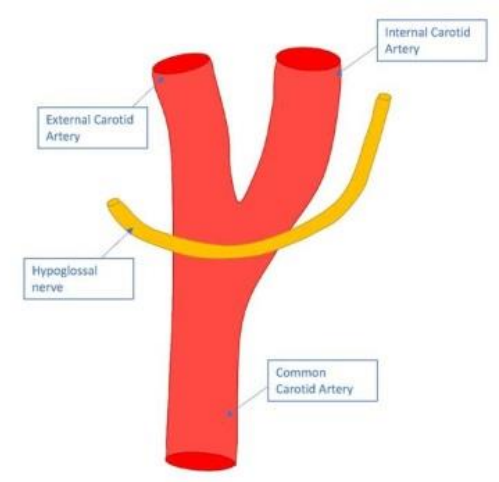

Figure 9: CNXII crosses the internal and external carotid arteries before moving anteriorly to enter the tongue.

Although it is rare, the aneurysmal dilatation of a dissection can compress this nerve resulting in an isolated CNXII as seen in this case (Figure 10).

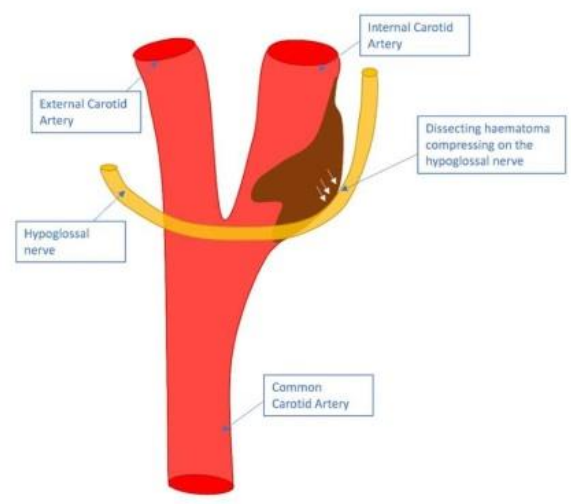

Figure 10: Aneurysmal dilatation of a dissection compressing CNXII.

Even though dissections are associated with major neck trauma, they can also be caused by trivial events like coughing, sneezing and hiccups [2]. Grau et al. postulate that there is a link with CAD and upper respiratory tract infections independent of coughing [3]. The combination of this patient's coughing and preceding coryzal illness are possible aetiologies for his dissection. 


\section{Learning Points/Take Home Messages}

1. CAD can present with ipsilateral facial pain, neck pain or with the sequalae of the dissection itself through distal embolisation of the intramural thrombus or through aneurysmal compression effects including Horner's syndrome and other lower cranial nerve palsies [1].

2. Most dissections occur spontaneously or after minor or trivial injury. There may be a link between recent infection and CAD that is not explained by the mechanical factors occurring during the infective process.

3. Although muscle atrophy develops over weeks or months, case reports have suggested that tongue atrophy from a CNXII palsy may develop within days.

\section{References}

1. National Institute for Health and Clinical Excellence. Stroke: The diagnosis and acute management of stroke and transient ischaemic attacks. Royal College of Physicians, London (2008).

2. Mathey DG, Wandler A, Rosenkranz M. Images in cardiovascular medicine. Hypoglossal-nerve palsy caused by carotid dissection. Circulation 121 (2010): 457.

3. Grau AJ, Brandt T, Buggle F, et al. Association of cervical artery dissection with recent infection. Arch Neurol 56 (1999): 851-856.

Citation: Muzzammil Ali, Yatin Sardana. Carotid Artery Dissection Causing an Isolated Hypoglossal Nerve Palsy. Archives of Clinical and Medical Case Reports 2 (2018): 129-134.

(C) 1 This article is an open access article distributed under the terms and conditions of the
Creative Commons Attribution (CC-BY) license 4.0

